\title{
Automatic Extraction of Mandibular Nerve and Bone from Cone-Beam CT Data
}

\author{
Dagmar Kainmueller ${ }^{1}$, Hans Lamecker ${ }^{2}$, Heiko Seim ${ }^{1}$, \\ Max Zinser ${ }^{3}$, and Stefan Zachow ${ }^{1}$ \\ 1 Zuse Institute Berlin, Germany \\ kainmueller@zib.de \\ 2 INRIA Sophia Antipolis, France \\ 3 Universitätsklinikum Köln, Germany
}

\begin{abstract}
The exact localization of the mandibular nerve with respect to the bone is important for applications in dental implantology and maxillofacial surgery. Cone beam computed tomography (CBCT), often also called digital volume tomography (DVT), is increasingly utilized in maxillofacial or dental imaging. Compared to conventional CT, however, soft tissue discrimination is worse due to a reduced dose. Thus, small structures like the alveolar nerves are even harder recognizable within the image data. We show that it is nonetheless possible to accurately reconstruct the $3 \mathrm{D}$ bone surface and the course of the nerve in a fully automatic fashion, with a method that is based on a combined statistical shape model of the nerve and the bone and a Dijkstra-based optimization procedure. Our method has been validated on 106 clinical datasets: the average reconstruction error for the bone is $0.5 \pm 0.1 \mathrm{~mm}$, and the nerve can be detected with an average error of $1.0 \pm 0.6 \mathrm{~mm}$.
\end{abstract}

\section{Motivation and Contributions}

Three-dimensional (3D) imaging has become an important technology for diagnosis and planning in dentistry and maxillofacial surgery [1. Cone beam computed tomography (CBCT) yields an alternative to conventional CT because of its affordable costs as well as its reduced dose per examination. Thus, CBCT is likely to become a preferred imaging technique for dental practices. One major application for CBCT is dental implantology. Here, a primary concern is an optimal and stable placement of implants within the jaw bone without any impairment of the facial nerves. As a side effect of the low dose, however, the signal to noise ratio is not that high as with CT and soft tissue structures cannot be discriminated clearly. In addition the field of view (FoV) is small compared to conventional CT. This renders the exact localization of the mandibular nerve canal within the alveolar bone highly challenging.

Stein et al. 2 present a Dijkstra and balloon inflation based method for interactively segmenting the nerve canal in CT data, yet report only qualitatively good accordance on five datasets. Hanssen et al. 3 suggest a level-set approach for interactive 3D segmentation of the nerve canals in CBCT data, but do not 
present any quantitative validation. Rueda et al. 4] propose a semi-automatic system to perform 2D segmentation of the lower cortical and trabecular bone as well as to detect the nerve canal and center in specific 2D slices of conventional CT data. Their method is based on an active appearance model, and requires manual initialization. It yields an accuracy of $1.6 \mathrm{~mm}$ for the cortical bone, and $3.4 \mathrm{~mm}$ for the dental nerve on 215 single 2D slices in the CT data. More recently, Yau et al. 5] proposed a semi-automatic method to segment the nerve canal from conventional CT data. It requires the user to manually specify a seed point for a subsequent automatic adaptive region-growing approach in consecutive slices of the CT data. However, no quantitative validation was performed.

In contrast to existing work, our method is based on (1) 3D segmentation of the complete mandibular bone surface and (2) localization of the $3 \mathrm{D}$ course of the mandibular nerve, both in a fully automatic manner. Instead of relying on conventional CT data, our method operates on CBCT data, an imaging modality increasingly used in clinical routine. Our approach yields an accuracy that significantly surpasses the $2 \mathrm{D}$ results of Rueda et al. 4]. It is based on a combined statistical shape model (SSM) of the bone surface and the course of the nerve, which extends the work of Zachow et al. 6. In order to match the SSM to CBCT data we extend the work of Lamecker et al. [7] in two major ways: (1) We use a modified version of the algorithm presented in Seim et al. [8] to segment the mandibular bone surface. Here, we adapt image feature extraction to the characteristics of mandibular CBCT data. (2) We improve an initial reconstruction of the nerves' position derived from the SSM using a Dijkstra-based tracing algorithm tailored to the specific characteristics of CBCT data.

\section{Image Data}

CBCT scanners aim for a compromise between image quality and dose, and hence produce images of lesser quality than conventional CT. For our study 106 datasets of complete mandibles were availible from a PACS at the University Hospital of Cologne, Germany, all of which acquired with a Sirona Galileos CBCT at the maxillofacial surgery department (patients of age 16 to 71, 56 female, 50 male). CBCT imaging is performed routinely in cases of suspected orbital floor fractures, mandibular condyle evaluation, wisdom teeth removal, abscesses, etc. Images are taken in seated position with a scan duration of about 15 seconds. All images consist of $512^{3}$ voxels with an isotropic voxel size of $0.3 \mathrm{~mm}$. The FoV is approx. $15 \mathrm{~cm}^{3}$. The X-ray source is operated at $85 \mathrm{kV}$ with a tube current of 5-7 mA. Fig. 1 right, depicts typical slices through such data. In each dataset the bone as well as the nerve canal were interactively labelled by an experienced dentist using the Amira software.

\section{Statistical Shape Model}

The SSM is generated on the basis of the CBCT datasets described in Sec. 2 For each mandibular bone a surface model is generated from the labellings, while for 

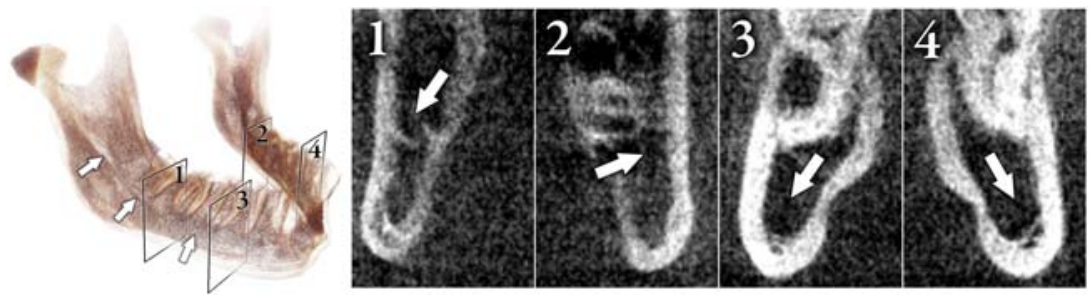

Fig. 1. Volume rendering (left) and coronal slices (right) of CBCT data. Arrows indicate the location of the nerve canal.

(a)

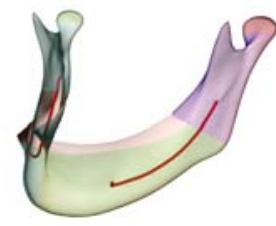

(b)

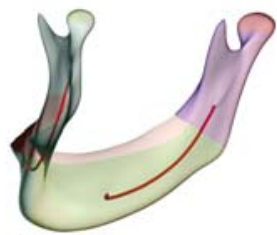

(c)

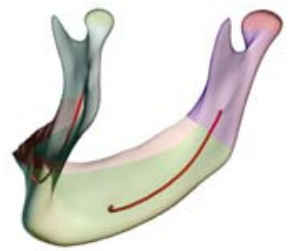

Fig. 2. SSM of mandible bone and nerves: mean shape (b), 1st mode of variation (a/c)

each pair of nerve canals piecewise linear center lines are computed using a skeletonization algorithm 9. To create the point distribution model underlying the SSM, correspondence must be established between both the bone surfaces and the nerve lines. We use the method of consistent patch decomposition and parameterization to create surface correspondences, see [7] for details. The mandibular surface model is divided into eight patches (Fig. 2) that are bounded by characteristic feature lines, detectable on every mandible. The teeth are not considered in the SSM due to an individually varying dentition state. For the nerves, we consistently resampled the center lines of the segmented nerve canals with a fixed number of points equally spaced starting at the mental foramen. After resampling all 106 mandibular shapes with a common reference mesh (triangulation for bone surface, piecewise linear segments for nerve) and aligning them in a common frame of reference, Principal Component Analysis (PCA) is performed. The SSM is then represented as a bi-linear function $S(b, T)=T\left(\bar{v}+\sum_{k} b_{k} \cdot p_{k}\right)$ of the weights $b_{k}$ of the PCA-eigenmodes $p_{k}$, and a global affine transformation $T$ of the coordinates. $\bar{v}$ is the average shape vector, whose dimension is three times the number of bone (8561) and nerve (200) points.

\section{SSM-Based Reconstruction of Bone and Nerve}

The SSM based method for reconstruction of the mandibular bone and nerves in CBCT data is composed of a preprocessing of the image data with a $3 \mathrm{D}$ median filter, a position initialization and a subsequent image driven adaptation of the SSM described in Sec. 3. We adapted an approach for pelvis segmentation [8] to 
the task of mandible segmentation. Note that only the mandible bone is adapted to image features, while the mandibular nerves are derived from the SSM.

Initialization. The pose initialization of the mandible SSM in CBCT data, that is based on the Generalized Hough Transform (GHT), closely follows a global approach for 3D object detection introduced by Khoshelham [10].

Image Driven SSM Adaptation. Segmentation using the SSM is the task of iteratively finding transformation and shape parameters $(b, T)$ such that the shape $S(b, T)$ approximates the unknown target shape $R^{*}$ as good as possible. Let $R^{i}=S\left(b^{i}, T^{i}\right)$ denote the segmentation in iteration $i$ : A displacement vector field $\Delta R^{i}$ is computed that assigns a vector $\Delta r_{j}$ to each vertex $j$ of $R^{i} . \Delta R^{i}$ describes the desired deformation of the model towards $R^{*}$ in the underlying image data $I$. Then, both transformation parameters $T$ and shape parameters $b$ are adapted to the target shape $\left(R^{i}+\Delta R^{i}\right)$, as originally proposed by Cootes et al. [1]. The following paragraph explains how $\Delta R^{i}$ is generated.

Image Features. The displacement vector field $\Delta R$ for surface $R$ is computed by analyzing $1 \mathrm{D}$ intensity profiles in the image data $I: \mathbb{R}^{3} \rightarrow \mathbb{R}$ : For each vertex $j$ of $R, I$ is sampled over a length $L$ along the surface normal $u_{j}$ at vertex position $v_{j}$. A cost function $c_{j}: P_{j} \rightarrow \mathbb{R}_{0}^{+}$is computed on the set of sampling points $P_{j}=\left\{v_{j}^{n}:=v_{j}+\left(\frac{n-1}{N_{j}-1}-0.5\right) \cdot L \cdot u_{j}: 1 \leq n \leq N_{j}\right\}$. The displacement vector at vertex $j$ is then defined as $\Delta r_{j}=v_{j}^{*}-v_{j}$, with $v_{j}^{*}=\operatorname{argmin}_{v_{j}^{n}} c_{j}\left(v_{j}^{n}\right)$. Dropping the indices for clarity, we define the cost at a sampling point $c(v)=$

$$
\left\{\begin{array}{l}
(2 i+1)\left(\frac{-g}{d I(v)}+2 \frac{|N-n|}{N}\right) \text { if } I(v) \in[t+i w, t+(i+1) w] \wedge d I(v)<-g, i=0,1,2 \\
7\left(\frac{-g}{d I(v)}+2 \frac{|N-n|}{N}\right) \text { if } I(v) \in[t, t+3 w] \text { and }-g<d I(v)<-0.5 \cdot g \\
30+2 \frac{|N-n|}{N} \text { else. }
\end{array}\right.
$$

Here, $t$ and $w$ define an intensity threshold and window width, and $g$ a threshold for gradient magnitude. $d I(v)$ denotes the directional derivative of $I$ along $u$.

\section{Dijkstra-Based Optimization of Nerve Reconstruction}

SSM-adaptation as described in Sec. 4 yields an accurate reconstruction of the mandible bone, as well as approximate nerve reconstructions. The SSM-based nerve reconstructions are not based on any image features, but are merely derived by the SSM. We use them as initialization for a Dijkstra-based optimization method. The SSM-based bone reconstruction is also utilized by excluding the area outside the reconstructed bone from the search space for the nerve. The key idea of our method is to build a graph through which the path with minimal cost from source to target is basically the "darkest tunnel" through the image data, while regions where a dark tunnel is surrounded by a brighter border are of particular interest. To achieve this, a graph with weighted edges is built based on the approximate nerve reconstruction as described in the following. Note that all indices used for graph description start at 1, unless stated otherwise. 


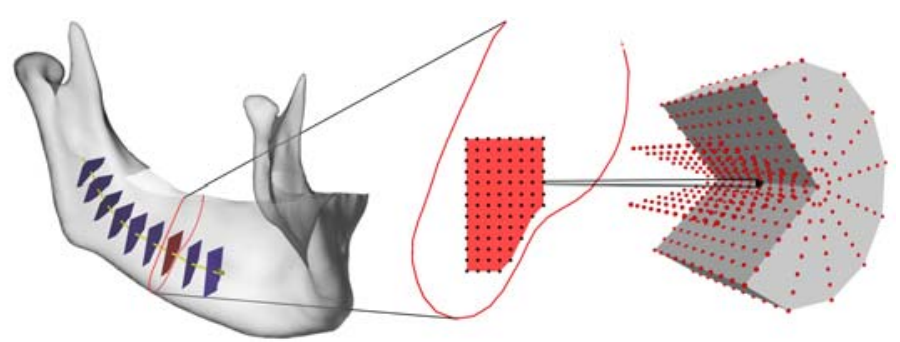

Fig. 3. Dijkstra Optimization. (left) Normal planes along initial nerve reconstruction. (middle) Graph nodes on a normal plane. (right) Sampling cylinder at a graph node.

Graph Nodes. For each point $\boldsymbol{p}_{k}$ at index $k$ of the piecewise linear initial nerve representation, equidistantly distributed points in the normal plane at $\boldsymbol{p}_{k}$ serve as graph nodes. Fig. 3 shows some exemplary normal planes (left) and a normal plane with graph nodes (middle). The normal plane at $\boldsymbol{p}_{k}$ is spanned by two directions perpendicular to the line tangent $\boldsymbol{t}_{k}$, namely $\boldsymbol{y}_{k}=\boldsymbol{t}_{k} \times \boldsymbol{x}_{\text {data }}$, where $\boldsymbol{x}_{\text {data }}$ is the X-axis of the image data coordinate system, and $\boldsymbol{x}_{k}=\boldsymbol{y}_{k} \times \boldsymbol{t}_{k}$. A graph node is described by $k$ and its indices $i, j$ on the normal plane. Let $N_{i}$, $N_{j}$ be the number of nodes and $X, Y$ the lengths for which the normal plane is considered in $x_{k^{-}}$and $y_{k^{-}}$direction, respectively. The position of node $(k, i, j)$ is then $\boldsymbol{p}_{k, i, j}=\boldsymbol{p}_{k}+\left(\frac{i-1}{N_{i}-1}-0.5\right) \cdot X \cdot \boldsymbol{x}_{k}+\left(\frac{j-1}{N_{j}-1}-0.5\right) \cdot Y \cdot \boldsymbol{y}_{k}$. In addition to these nodes, two "artificial" nodes serve as source and target of the graph.

Graph Edges. The graph contains directional edges from each node $(k, i, j)$ to all nodes $(k+1, i+d i, j+d j)$ with $d i, d j \in\{-1,0,1\}$, as well as directional edges from the source to all nodes with $k=1$, and from all nodes with $k=N$ to the target, where $N$ is the number of points on the nerve representation.

Edge Weights. For any edge starting at node $(k, i, j)$, a scalar cost function $c$, evaluated at position $\boldsymbol{p}_{k, i, j}$, serves as edge weight. For edges starting at the source node, the edge weights are 0 . The cost $c\left(\boldsymbol{p}_{k, i, j}\right)$ is computed from intensities sampled inside a cylinder with center $\boldsymbol{p}_{k, i, j}$, orientation $\boldsymbol{t}_{k}$, some length $H$ and radius $R$. Fig. 3 (right) shows an exemplary cylinder with its sampling points. A sampling point is described by a length index $h$, a radius index $r$, and an angle index $a$. Let $N_{h}$ be the number of sampling points in length direction, $N_{r}$ the number of sampling points along a radius, and $N_{a}$ the number of angles for which radii are sampled. Then the position of sampling point $(h, r, a)$ is $\boldsymbol{p}_{0}+\frac{h-1}{N_{h}-1} \cdot H \cdot \boldsymbol{t}_{k}+\frac{r-1}{N_{r}-1} \cdot R \cdot \boldsymbol{r}_{a}$ with $\boldsymbol{r}_{a}$ being a normalized radius vector, rotated by an angle $\frac{a-1}{N_{a}-1} * 2 \pi$ around $\boldsymbol{t}_{k}$, and $\boldsymbol{p}_{0}=\boldsymbol{p}_{k, i, j}-0.5 \cdot H \cdot \boldsymbol{t}_{k}$. Note that for $r=1$, no angle index is necessary to describe the sample point.

To determine the cost $c\left(\boldsymbol{p}_{k, i, j}\right)$, the unfiltered image intensities at the cylinder sampling points are evaluated as follows: The mean "inner" intensity $m i$ and standard deviation $s i$ is computed from all sampling points with $r$ no bigger than an "inner radius index" $r_{i}$. For each angle index $a$, the mean "border" intensity $m b_{a}$ and standard deviation $s b_{a}$ is computed from all sampling points 


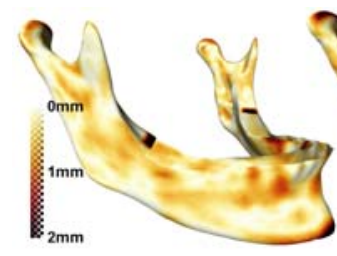

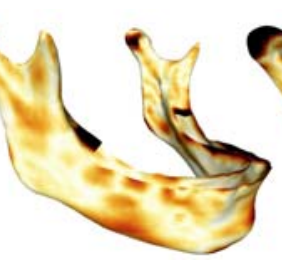

(a)

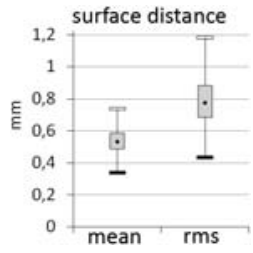

(b)

Fig. 4. Surface reconstruction with statistical shape model. (a) Color encodes distance to gold standard surface. from left to right: good, average and bad case. (b) Average surface distance error metrics.

with $r>r_{i}$ and $r$ no bigger than a "border radius index" $r_{b}$. Furthermore, the mean "outside" intensity $m o_{a}$ is computed for each angle index a from all sampling points with $r>r_{b}$. The number $c f$ of angle indices is counted for which $m b_{a}-0.1 \cdot s b_{a}>m i+s i$ and $m b_{a}-0.1 \cdot s b_{a}>m o_{a}$. Then the cost is $c\left(\boldsymbol{p}_{k, i, j}\right)=m i-50 \cdot c f$. If a graph node position $\boldsymbol{p}_{k, i, j}$ lies outside the mandibular bone as reconstructed by the SSM, the cost is set to infinity.

\section{Results}

We evaluated the SSM based reconstruction as well as the Dijkstra based optimization (OPT) described in Sec. 4 and 5 on the 106 CBCT datasets described in Sec. 2. For each dataset, before performing SSM based reconstruction, the respective training shape was removed from the mandible SSM described in Sec. 3, i.e. the evaluation was conducted in a leave-one-out manner. The respective training shape that has been left out serves as gold standard reconstruction for both bone and nerves.

From experiment, we set our method's parameters as follows: SSM adaptation: consider 80 shape modes. Image features: $L=6 \mathrm{~mm}, t=350, w=180, g=$ 150/mm. Optimization, graph nodes: $X=12 \mathrm{~mm}, Y=7 \mathrm{~mm}, N_{i}=121, N_{j}=71$, cylinder: $L=3 \mathrm{~mm}, R=2.1 \mathrm{~mm}, N_{l}=11, N_{r}=8, N_{a}=12, r_{i}=4, r_{b}=6$.

The average errors for the mandible surface reconstructions as compared to the respective gold standard surfaces are: mean, root mean square (rms) and maximum surface distance: $0.5 \pm 0.1 \mathrm{~mm}, 0.8 \pm 0.2 \mathrm{~mm}$, and $6.2 \pm 2.3 \mathrm{~mm}$, see also Fig. 4(b). Fig. 4 (a) shows exemplary reconstructions and their distances to the respective gold standard surface. Apart from errors around the teeth, the largest errors occur at the mental protuberance and the condyles, due to the increasing noise towards the fringe of the field of view. The average mean curve distances for the SSM based nerve reconstructions are $1.7 \pm 0.7 \mathrm{~mm}$ (right nerve), and $2.0 \pm 0.8 \mathrm{~mm}$ (left nerve).

For the optimized nerve reconstructions, the average mean curve distances to the respective gold standard nerve are $1.0 \pm 0.6 \mathrm{~mm}$ (right nerve), and $1.2 \pm$ $0.9 \mathrm{~mm}$ (left nerve). The average fraction of the optimized reconstruction that lies within the gold standard nerve canal is $80 \pm 24 \%$ (right nerve), and $74 \pm$ 

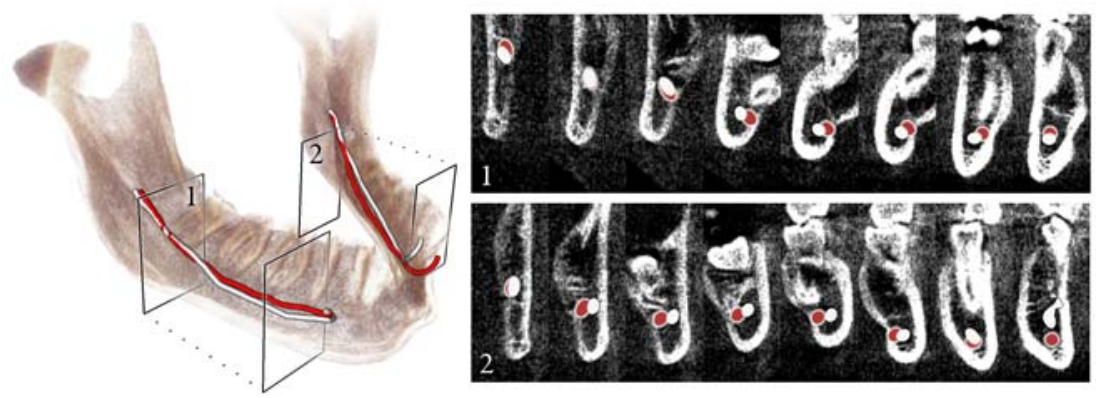

Fig. 5. Reconstruction by optimization, exemplary case for a bad reconstruction. White line: gold standard nerve. Red line: reconstructed nerve. Error measures for this case: Mean distance to gold standard nerve: right $1.5 \mathrm{~mm}$, left $2.1 \mathrm{~mm}$. Fraction that lies within the gold standard nerve canal: right $45 \%$, left $30 \%$.

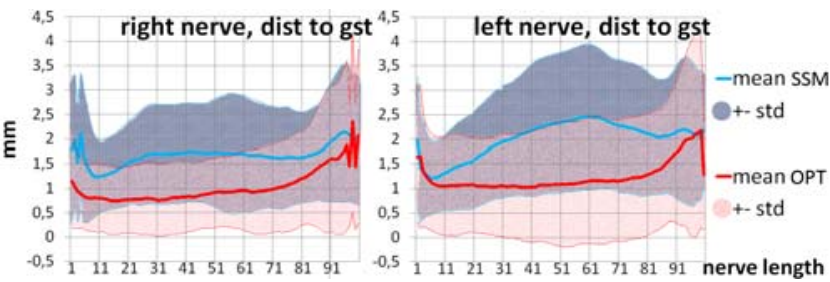

(a)

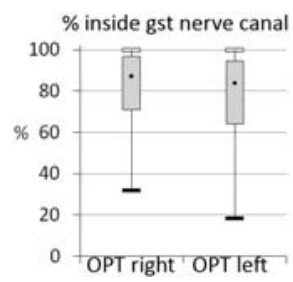

(b)

Fig. 6. (a) Average reconstruction error (SSM and OPT) along the nerve from posterior to anterior end (1..100). (b) Nerve fraction within gold standard nerve canal.

27\% (left nerve), see also Fig. 6(b). Fig. 5] shows an exemplary optimized nerve reconstruction with high reconstruction error. Fig. 6)(a) shows the average curve distance of SSM based and optimized nerve reconstructions per point along the curve. This illustrates that the optimization method is able to reduce the reconstruction error significantly in a region in the middle of each nerve, while the reduction is not that obvious towards the ends of each nerve.

\section{Conclusions and Future Work}

We presented an accurate and robust method to automatically reconstruct a geometric 3D model of the mandible including the course of the alveolar nerve from CBCT data. There is still room for improvement of the nerve reconstruction, especially concerning the ends of the nerve canal. In this work we chose a conceptually simple approach for nerve detection, yet other methods for tracing tubular structures may be considered, e.g. as described for vessel detection, see for instance [1213] and references therein.

In future work, the statistical shape model shall be extended to distinguish cortical and trabecular bone as well. Furthermore, a mid-term goal is to find a 
way to incorporate the teeth, too. This is more challenging since the number of teeth may vary between patients, especially in clinical cases, and it is not quite clear how to incorporate topological changes into the model.

Acknowledgments. D. Kainmueller is funded by the DFG collaborative research center SFB 760.

\section{References}

1. Schramm, A., Rücker, M., Sakka, N., Schoen, R., Dueker, J., Gellrich, N.C.: The use of cone beam CT in cranio-maxillofacial surgery. In: Lemke, H., Inamura, K., Doi, K., Vannier, M., Farman, A. (eds.) CARS. Int. Congress Series, vol. 1281, pp. 1200-1204. Elsevier, Amsterdam (2005)

2. Stein, W., Hassfeld, S., Muhling, J.: Tracing of thin tubular structures in computer tomographic data. Computer Aided Surgery 3, 83-88 (1998)

3. Hanssen, N., Burgielski, Z., Jansen, T., Lievin, M., Ritter, L., von Rymon-Lipinski, B., Keeve, E.: Nerves - level sets for interactive 3d segmentation of nerve channels. In: ISBI, pp. 201-204 (2004)

4. Rueda, S., Gil, J.A., Pichery, R., Raya, M.A.: Automatic segmentation of jaw tissues in CT using active appearance models and semi-automatic landmarking. In: Larsen, R., Nielsen, M., Sporring, J. (eds.) MICCAI 2006. LNCS, vol. 4190, pp. 167-174. Springer, Heidelberg (2006)

5. Yau, H.T., Lin, Y.K., Tsou, L.S., Lee, C.Y.: An adaptive region growing method to segment inferior alveolar nerve canal from $3 \mathrm{~d}$ medical images for dental implant surgery. Computer-Aided Design and Applications 5(5), 743-752 (2008)

6. Zachow, S., Lamecker, H., Elsholtz, B., Stiller, M.: Is the course of the mandibular nerve deducible from the shape of the mandible? Int. J. Computer Assisted Radiology and Surgery 1(1), 415-417 (2006)

7. Lamecker, H., Zachow, S., Wittmers, A., Weber, B., Hege, H.C., Elsholtz, B., Stiller, M.: Automatic segmentation of mandibles in low-dose CT-data. Int. J. Computer Assisted Radiology and Surgery 1(1), 393-395 (2006)

8. Seim, H., Kainmueller, D., Heller, M., Lamecker, H., Zachow, S., Hege, H.C.: Automatic segmentation of the pelvic bones from CT data based on a statistical shape model. In: EG Workshop on VCBM, Delft, Netherlands, pp. 93-100 (2008)

9. Sato, M., Bitter, I., Bende, M., Kaufman, A., Nakajima, M.: TEASAR: Treestructure extraction algorithm for accurate and robust skeletons. In: Barsky, B.A., Shinagawa, Y., Wang, W. (eds.) Proc. Pacific Graph. Conf. on Comp. Graph. and Application, pp. 281-289. IEEE, Los Alamitos (2000)

10. Khoshelham, K.: Extending generalized hough transform to detect $3 \mathrm{~d}$ objects in laser range data. In: Rönnholm, P., Hyyppä, H., Hyyppä, J. (eds.) Proc. ISPRS Workshop Laser Scanning and SilviLaser. IAPRS, vol. 36, pp. 206-211 (2007)

11. Cootes, T.F., Taylor, C.J., Cooper, D.H., Graham, J.: Active Shape Models - Their Training and Application. Comput. Vis. Image Underst. 61(1), 38-59 (1995)

12. Wong, W.C., Chung, A.C.: Probabilistic vessel axis tracing and its application to vessel segmentation with stream surfaces and minimum cost paths. Medical Image Analysis 11(6), 567-587 (2007)

13. Poon, K., Hamarneh, G., Abugharbieh, R.: Live-vessel: Extending livewire for simultaneous extraction of optimal medial and boundary paths in vascular images. In: Ayache, N., Ourselin, S., Maeder, A. (eds.) MICCAI 2007, Part II. LNCS, vol. 4792, pp. 444-451. Springer, Heidelberg (2007) 